\title{
Histological, radiological and clinical outcomes of sinus floor elevation using lateral approach pre-/post- extraction of the severe periodontally compromised maxillary molars: a split-mouth randomized clinical trial
}

\section{Zhaoguo Yue}

Peking University Hospital of Stomatology

Qi Liu

BYBO Dental Hospital

\section{Haidong Zhang}

Peking University School and Hospital of Stomatology

Jingwen Yang

Peking University School and Hospital of Stomatology

Yalin Zhan

Peking University School and Hospital of Stomatology

Ye Han

Peking University School and Hospital of Stomatology

Yue Yan

Peking University School and Hospital of Stomatology

Lili Miao

Peking University School and Hospital of Stomatology

Wenxue Huang

Peking University School and Hospital of Stomatology

Churen Zhang

Peking University School and Hospital of Stomatology

Jianxia Hou ( $\nabla$ jxhou@163.com )

Peking University School and Hospital of Stomatology

Study protocol

Keywords: Dental implants, maxillary sinus floor elevation, lateral approach, Bone grafting, periodontitis

Posted Date: July 19th, 2019 
DOI: https://doi.org/10.21203/rs.2.11722/v1

License: (c) (1) This work is licensed under a Creative Commons Attribution 4.0 International License. Read Full License 


\section{Abstract}

Background Residual alveolar bone volume is a critical factor for the dental implants' survival [1]. When the volume of alveolar bone in the posterior maxillary region is less than $4 \mathrm{~mm}$, the maxillary sinus floor elevation (MSFE) with lateral approach is an effective way to solve this problem [2]. Traditionally, this standard approach is usually delivered 4 months after the extraction of the tooth. However, the defective dentition due to the extraction would cause impaired masticatory function during the long period of the bone regeneration, especially when molars on both sides are severely periodontal compromised and need to be extracted. So, as periodontists, we start to wonder if we can maintain a compromised tooth so that we perform the sinus floor elevation before the extraction (the modified MSFE) to take fully advantage of residual strength of the teeth. Methods/Design The proposed study is designed as a prospective singlecenter, split-mouth design, randomized controlled trial. We plan to recruit 10 patients whose molars on both sides are severely periodontal compromised and in need of dental implant treatment in the atrophied posterior maxilla. The two sides of each patients will be divided into two groups randomly using Randomizer for Clinical Trials (Institute for Medical Informatics, Statistics and Documentation, Medical Univ. Graz). Test group: sinus floor augmentation before tooth extraction (the modified MSFE); Control group: sinus floor augmentation after tooth extraction (the standard MSFE). Implant survival rates, mucosal conditions around the implants, complications, treatment time and postoperative discomfort (visual analogue scale for intraoperative pain and postoperative pain/discomfort) will be recorded. The volume of the alveolar bone will be measured $6,12,18$, and 24 months after the MSFE surgery by means of CBCT. Histological and histomorphometic analysis will be performed on biopsies retrieved from both side of grafting sites. Discussion The current study will explore the effectiveness of the modified MSFE on minimizing the discomfort period caused by defected dentition. To determine whether the modified MSFE will promote the grafting outcomes, the quality and the quantity of the osteogenesis on the sinus floor will also be compared. Depending on the result of the current study, we will determine all the protocols and details about the modified MSFE, including indications, detailed methods, post-operational complications and managements.

\section{Background}

When choosing a proper way to restore the impaired dentition, dental implants can be a great option. For the atrophy posterior maxilla with the residual bone height less than $4 \mathrm{~mm}$, it is wise to conduct the maxillary sinus augmentation surgery to create an optimal bone condition before the implantation [2].

In the 1970s, Tatum and his colleagues first proposed utilizing maxillary sinus to increase available alveolar bone volume [3]. As the procedure was improved in 1986 [4], the principles and details of the maxillary sinus augmentation using lateral approach became recognized.

As it is generally known, maxillary sinus augmentation can be performed transalveolarly or with lateral approach. Comparing to transalveolar approach, the maxillary sinus augmentation with lateral approach provides a direct view of the surgical field, minimizes the risk of perforation, and guarantees the volume 
of grafted bone material $[2,22,23]$. However, multiple intra- or post-operative complications and the discomfort caused by this procedure are still problems that need to be tackled [21]. In particular, the impaired function during the healing time has drawn a lot of attention, and experts have put forward different methods to improve the traditional procedure $[6,7,8,9,10]$. Unfortunately, all previous researches concentrated on the method of shortening the healing time instead of directly improving the masticatory function. Therefore, the discomfort following impaired oral function, especially for patients whose molars on both sides are extracted due to severe periodontitis, would remain and last for at least 4-7months [16]. In order to improve these patients' quality of life during the osteogenesis period, it is reasonable to ask: Is it better to extract the compromised teeth after the sinus floor is augmented?

In 2011, a clinical trial carried out by Graetz C et al. [24] indicated that, although being treated actively, $40 \%$ of 'hopeless' teeth do not survive for longer than 4 years on average. Based on this and the high success rate of dental implants nowadays, earlier strategic extractions of hopeless teeth are considered to be beneficial for the implant placemen [18]. However, the exact date of the extraction is very critical. As it is proved in previous studies [19], it is also necessary to fully utilize and extend the capacity of the natural tooth. Given that the integrity of the dentition is fundamental to high masticatory efficiency [20], optimizing the longevity of the dentition will improve the patients' quality of life.

Recently, in a clinical trial involving 65 patients, extended maxillary sinus augmentation (EMSA) to the apical area of the neighboring teeth was proposed by llan Beitlitum et.al [11]. As the reliability and the safety of EMSA were proved in this trail, this new approach enables future implant placement while avoiding the need for sinus reentry and proximal teeth extraction. Thus, sinus floor augmentation with corresponding tooth on site should be considered feasible to some extent.

In order to fix the impaired oral function of the periodontally compromised patients whose molars on both sides need to be extracted, the current research proposes the maxillary sinus floor elevation with lateral approach before the tooth extraction. We try to maintain the compromised tooth so that we perform the sinus floor elevation before the extraction to take fully advantage of residual function of the tooth. Thus, the dentition-impaired period will be reduced by at least 6 months. The extraction and the implantation will also be done in one visit to minimize the number of surgery. With this new procedure, we look forward to reducing the discomfort caused by surgical interventions or impaired function to some extent. At the same time, it will be a good chance for researchers to investigate the promoting effect of functional stimulation on bone regeneration. We expect to take advantage of the functional stimulation at the site to improve the outcome of bone regeneration after the grafting, which may lead to a better condition for the simultaneously implantation following the extraction later.

\section{Objective and hypotheses}

The aim of current study is to compare the outcomes of traditional maxillary sinus floor elevation (MSFE) before tooth extraction with that of the traditional approach, in terms of patient satisfaction and the comparison of the outcomes of bone grafting both histologically and radiologically. 
The primary hypotheses are (1) the modified MSFE before the tooth extraction could achieve ideal clinical outcomes similar to the traditional MSFE but with less patient discomfort and (2) the modified MSFE before the tooth extraction could also achieve better outcomes of osteogenesis both histologically and radiologically. Considered outcomes include implant survival rate, complications, marginal bone remodeling, patient-reported outcome (visual analog scale, or VAS). Histologic and radiological analyses of bone tissue will also be considered.

\section{Methods}

\section{Overview}

The proposed study is designed as a prospective single-center, split-mouth design, randomized controlled trial. We plan to recruit 10 patients whose molars on both sides are severely periodontally compromised and in need of dental implant treatment in the atrophied posterior maxilla. The participants will be recruited by the research staff from Peking University Hospital of Stomatology (Beijing, China), and all procedures, recalls, and analysis will also be conducted at this hospital. The study has been approved by the ethics committee of the Peking University School and Hospital of Stomatology (PKUSSIRB201840191). The study has been registered on International Clinical Trails Registry Platform under identifier number ChiCTR1900020648.

The systematic health condition of all the participants must be recognized and qualified to undergo the series of treatment. All patients joining the study will receive standard periodontal initial therapy and periodontal maintenance at regular intervals. Only if the patient is clinically periodontal healthy should we begin to perform the maxillary sinus floor augmentation surgery. Before the surgery, all patients will receive a clinical and radiographic assessment. Cone beam computed tomography (CT) scan will be taken to assess the initial bone height, bone quality, and alveolar width.

\section{Inclusion criteria}

1. Patients with bilateral first/second molars that need to be extracted due to severe periodontal disease (i.e. the prognosis of which is regarded as 'hopeless'[25]) and need to be replaced by implants;

2. Residual alveolar bone height of the compromised teeth is between $1-4 \mathrm{~mm}$;

3. Patients who are above 18 years old;

4. Patients with optimal oral hygiene and good compliance;

5. Patients with healthy maxillary sinuses;

6. Non-smokers; 
7. The general health of patient is suitable for implant placement and sinus floor elevation;

8. The patient who is willing to sign the informed consent and follow the experimental follow-up rules.

\section{Exclusion criteria}

1. Residual alveolar bone height of the compromised teeth is more than $4 \mathrm{~mm}$ or less than $1 \mathrm{~mm}$;

2. Patients who are under 18 years old;

3. Patients with poor oral hygiene and/or active periodontitis;

4. Patients who are suffering from rhinitis, sinusitis; or rather large cyst is found in the maxillary sinus;

5. Smokers;

6. Patients who is suffering uncontrolled systemic diseases such as diabetes, cardiovascular diseases, immune system diseases, severe osteoporosis and so on;

7. Patients who are NOT willing to sign the informed consent and who refuse to follow the experimental follow-up rules.

\section{Recruitment}

Recruitment will be performed in the Peking University Hospital of Stomatology. Eligible patients who are willing to participate have the right to know all the details of the study. They cannot be included in the current research until they sign the consent forms. Figure 1 shows the flow of participants through the trial. Figure 2 is the SPIRIT figure of the current study.

\section{Groups, randomization, and blinding}

The two sides of each patient will be divided into two groups randomly using Randomizer for Clinical Trials (Institute for Medical Informatics, Statistics and Documentation, Medical Univ. Graz) which will allocate each side a number: Group 1 (test group): Sinus floor augmentation before tooth extraction; Group 2 (control group): Sinus floor augmentation after tooth extraction. In accordance with the principle of blindness, the surgeon will have no access to the statistical analysis process. Similarly, the interventions and grouping will be concealed from the statistical analyst.

\section{Surgical interventions}


Implant and bone substitute: All the patients will receive Straumann BL implants with SLA surface (Straumann AG, Basel, Switzerland). The bone substitutes are Deproteinized Bovine Bone Mineral (Biooss ${ }^{\circledR}$, Geistlich, Wolhusen, Switzerland)

\section{Maxillary sinus augmentation}

For the test group, except for a few specific changes, the surgical procedure will be largely referred to as the Tatum's method [12]. The sinus lift procedure will be performed before the extraction of the compromised tooth/teeth. At first, we will treat these teeth actively to eliminate the inflammation of the periodontal tissue. After the periodontal status of the compromised teeth is maintained to a clinically healthy degree (Plaque Index $\leq 1$; NO MORE bleeding on probing; Probe depth $\leq 4 \mathrm{~mm}$;), a crevicular incision will be performed, which is designed to be extended to at least one adjacent tooth both mesially and distally. A full-thickness access flap will be prepared, and an access window $(5 \mathrm{~mm} \times 8 \mathrm{~mm})$ will be created on the lateral wall of the maxillary sinus, using a round diamond bur under irrigation with sterile saline. The lower border of the window must be at least $3-5 \mathrm{~mm}$ above the floor to keep it away from the teeth on the site. The sinus membrane will be raised up for at least $10 \mathrm{~mm}$, exceeding the apical range of the tooth (both buccolingually and mesiodistally). And the generated cavity within the maxillary sinus will be filled with Deproteinized Bovine Bone Mineral (Bio-oss ${ }^{\circledR}$, Geistlich, Wolhusen, Switzerland), After the bone substitute is placed, a resorbable collagen membrane (Bio-Gide®, Geistlich, Wolhusen, Switzerland) will be placed to cover the antrostomy defect. Interrupted sutures or mattress sutures will be performed using PROLENE monofilament non-absorbable sutures (Ethicon, Johnson \& Johnson International) to achieve primary flap closure. All patients will receive preoperative antibiotic prophylaxis, consisting of $500 \mathrm{mg}$ amoxicillin 3 times daily and for 7 days postoperatively. After a healing period of 6 months (prior to dental implant placement), a cone beam computed tomography (CBCT) scan will be taken to observe the increase in vertical bone height at the planned dental implant sites.

For the control group, the procedure will be performed 3 months after the extraction of the compromised teeth/tooth. Besides, the traditional MSFE will be fully in accordance with the previously mentioned method introduced by Tatum. [12]

\section{Implant placement}

Six months after the MSFE procedure, dental implant surgery will be performed under local anesthesia. For the test group, the compromised tooth will be extracted with minimal invasion. After that, biopsy will be obtained with a hollow trephine drill with an internal diameter of $2 \mathrm{~mm}$ and a length of $6 \mathrm{~mm}$, and the sample will be copiously irrigated with sterile saline before it is fixated in phosphate-buffered formaldehyde. An implant hole will be prepared simultaneously, and the position and the orientation will 
be restoratively driven. Straumann dental implant with a diameter of $4.8 \mathrm{~mm}$, a length of $10 \mathrm{~mm}$, and a sand-blasted, large-grit, acid-etched surface will be insert in the planned site, with a depth where the platform reaches the bottom of the socket. Then the implant will be mounted with healing caps, and sutured with PROLENE monofilament non-absorbable sutures (Ethicon, Johnson \& Johnson International). To double-check the implant position, postoperation periapical radiographs using paralleling technique will be taken.

For the control group, a crestal incision will be made with mesial and distal buccal vertical release incisions. A full thickness mucoperiosteal flap will be raised to expose the alveolar ridge and the grafting site. Other steps will stay the same as described above.

For both sides, after 4 months of the unloaded implant healing, stage-two surgery will be performed under local anesthesia to replace the implant cover screws with the healing abutments. After another month, the crown will be placed.

\section{Outcomes}

\section{Baseline assessment}

Cone beam CT (СBCT) will be taken before and after the MSFE surgery. These digital radiographic data will be used as the baseline radiographic data. Treatment time will be calculated from incision to suture. Patients will be asked to give their answers regarding their intraoperative discomfort and postoperative pain levels immediately and two weeks after the surgery, using a 100-mm visual analogue scale (VAS) with "very dissatisfied" on the left and "very satisfied" on the right.[13]

\section{Bone biopsy/ Histology and Histomorphometric Analysis}

After an osteogenesis period of 6 months, histological and histomorphometic analyses will be performed on biopsies retrieved from grafting sites. The bone biopsies will be taken during dental implant surgery with a hollow trephine drill, fixated in $10 \%$ formaldehyde $\left(\mathrm{pH} 7.4 ; 4^{\circ} \mathrm{C}\right)$, transferred to $70 \%$ ethanol and stored until use for histomorphometric analysis. The sample will be subsequently dehydrated in descending concentrations of ethanol, embedded in low-temperature polymerizing methyl methacrylate, and sectioned ( $4 \mu \mathrm{m}$ thickness sections), and stained with both hematoxylin-eosin and modified Mallory aniline blue. The sections will be divided up into regions of interest (ROI). For each ROI, histomorphometric measurements will be performed blindly. The following parameters will be recorded: bone volume (BV), graft volume (GV), osteoid volume (OV), and connective tissue volume (CTV) will be 
calculated as a percentage of the total tissue volume (TV). Osteoid-graft perimeter (BGP) and connective tissue-graft perimeter (CTGP) will be calculated as a percentage of the total graft perimeter (GP)[14]

\section{Follow-up assessment}

All the included patients will be recalled for clinical and radiographic assessment at 6 (right before the implant placement), 12(right before the crown placement), 18, and 24 months after the MSFE surgery. VAS will be distributed to each participant at 6 and 12 months after the surgery to evaluate the overall satisfaction during the whole treatment period. Periapical radiographs will be taken to survey marginal bone level, immediately after the implant surgery, at the 6-month examination, and at the 1-year examination. We will conduct a paralleling technique when we acquire the radiographs for standardization. Radiographic analysis will be conducted by a CBCT analysis software (Mimics Research 18.0, Materialise, Belgium). Survival rates, and complications, if they occur, will also be documented. The proportion of implants being retained in the mouth will be regarded as the survival rate. The complications that will be recorded include hemorrhage, Schneider's membrane perforation, infection (especially at the grafting site), sinusitis, and other potential risks that cannot be anticipated before the intervention.

\section{Primary parameters of the trial}

The primary parameters of the current trial are implant survival rates, postoperative discomfort (pain), histological evaluation of a biopsy at implant site, and radiographic measurement of bone tissue.

\section{Secondary parameters}

The secondary parameters of this study include the followings:

1.The operation time of the maxillary sinus floor augmentation.

2. Complications during and after the surgery, including infection, hematoma, nasal bleeding, and Benign Paroxysmal Positional Vertigo (BPPV).

3.Mucosal condition around the implant: The parameters include depth of probing, sulcus bleeding (by

bleeding index), plaque around the restorations (by modified plaque index), and keratinized mucosa width will be documented. And the occurrence of biological complications (peri-implantitis and peri-implant mucositis) will be recorded.

\section{Sample size}


We use PASS 11.0(NCSS) to roughly estimate the sample size needed for the current study. The algorithm is based on the formula: $n=2(t a+t 1-\beta) 2 \sigma 2 / \delta 2$. The significance criteria are set to alpha $=0.05$ (error) and beta $=0.10$ (type II error). The analysis is two-tailed. To reach a power of $80 \%$, at least 6 sites should be distributed to each group. Assuming a dropout rate of $20 \%, 10$ sites in each group are needed: a total of 10 patients with 20 sites.

\section{Data collection and management}

All of the data will be documented in an exclusive computer, as well as in paper. Two experimenters will conduct the statistical analysis independently. To avoid the potential bias, only one random implant will be evaluated in patients who are treated with multiple implants in the posterior maxilla.

\section{Statistical analysis}

For any continuous data (such as residual bone height), the consistency between the two examiners will be tested by the intra-class correlation coefficient. For the descriptive data, the consistency will be tested by Cohen's k statistics. Descriptive statistics include frequency values (absolute and relative values) and metric data (arithmetic mean, standard deviation, and median). The chi-square t test will be applied to the evaluation of vertical bone gain in the test group vs. the control group. Histomorphometric measurements of the biopsies taken from both groups will be compared using nonparametric Wilcoxon t-test for paired samples. To determine the power, post-hoc Wilcoxon signed-rank test using Cohen's $d$ test will be performed. Statistical analysis will be performed using the statistical package SPSS 19.0 (Microsoft). P values will be two-sided and considered statistically significant if less than or equal to 0.05 .

\section{Missing data}

Taking possible loss into consideration, the proper sample size is estimated using a statistical analysis software package (PASS 11.0, NCSS, the U.S.A.). Moreover, we will account for other types of missing data by handling drop-outs as nonsuccess or nonsurvival, in accordance with the intention-to-treat principle.

\section{Ethical considerations}

\section{Ethical approval}

The study has been approved by the ethics committee of the School and Hospital of Stomatology, Peking University (PKUSSIRB-201840191). Eligible patients will receive the study information and consent forms. The patients who are not willing to sign the consent will not be recruited. 


\section{Withdrawal}

Before any interventions are performed on patients, they will be fully informed about the content and the risks of the current study. All participants have the right to withdraw from the research at any time for any reason. The required treatment will be performed whether they decide to participate in the current research or not.

\section{Dissemination of results}

The outcomes of the research will be recorded and published by professional editors in an international journal. For public access, an analysis of the study outcomes will be uploaded to Chictr.org.cn.

\section{Discussion}

As it is reviewed previously [11], the safety and the reliability of the maxillary sinus floor augmentation before the extraction of the corresponding tooth were fully proved. Despite of this, its effectiveness on reducing the discomfort during or after the operation is still uncertain, especially when both sides of molars are so periodontally compromised that need to be extracted.

With the MSFE before the extraction, we transfer delayed implantation after the MSFE to immediate implantation so that we could cut down the numbers of operations by at least 1-2 times. At the same time, maintaining the tooth on site will minimize the discomfort period caused by the defective oral function to at most 3 months. We hope this new arrangement of intervals will improve patient satisfaction with the procedure.

Since the loss of the available bone volume is caused by the resorption of the alveolar bone and the pneumatization of the sinus mainly due to the loss of the functional stimulation of the teeth [17], we presume in the current study that the functional stimuli will be beneficial to the outcomes of floor augmentation, both radiographically and histologically. The quality and the quantity of the osteogenesis will be also compared in the study to decide whether the hypotheses will be accepted.

As a result of the current study, we will finally determine all the protocols and details about the modified MSFE, including indications, detailed methods, post-operational complications and managements. 


\section{Challenges}

First of all, since we plan to elevate the Schneider's membrane with the tooth/teeth on site, it will be very difficult to keep the membrane intact during the whole lifting process, especially when the sinus floor is extremely near the apex of the root and, thus, full of 'lumps'. Evaluations including CBCT analysis should be carried out carefully before the procedure. Operators should be skilled, so that the membrane must be stripped totally along the contour of the floor. Fixing methods should be prepared beforehand as a backup plan in case perforation happens.

Secondly, the radiographic analysis is hard to be precise. Because of the complexity of the anatomic structures in the posterior maxilla, the exact border of the maxillary sinus floor in the image can be hard to identify. Therefore, in some cases, it would likely be difficult to calculate the amount of changing of the hard tissue precisely.

\section{Trial Status}

The trial has been registered at Chictr.org.cn and the recruitment is opened for the study. This is the $3^{\text {rd }}$ edition of the study protocol (2019-4-15). The date recruitment began: 2019-02-01. The approximate date when recruitment will be completed: 2021/02/01.

\section{Abbreviations}

MSFE: Maxillary sinus floor elevation; EMSA: Extended maxillary sinus augmentation; VAS: visual analog scale; CT: Computed tomography; СBCT: Cone beam computed tomography; PLI: Plaque Index; ROI: Regions of interest; BV: Bone volume; GV: Graft volume; OV: Osteoid volume; CTV: Connective tissue volume; TV: Total tissue volume; BGP: Osteoid-graft perimeter; CTGP: Connective tissue-graft perimeter; GP: Graft perimeter; BPPV: Benign Paroxysmal Positional Vertigo

\section{Declarations}

\section{Ethics approval and consent to participate}

The study plan and consent to participate were reviewed and approved by the ethics committee of the Peking University School and Hospital of Stomatology (PKUSSIRB-201840191). The study complies with the relevant Standard Protocol Items: Recommendations for Interventional Trials (SPIRIT) Checklist (Additional file 1). 


\section{Consent for publication}

Not applicable.

\section{Availability of data and materials}

The datasets generated and analyzed during the current study are available in the ResMan ${ }^{\circledR}$ research manager repository, http://www.medresman.org

\section{Competing interests}

The authors declare that they have no competing interests.

\section{Funding}

This trial is conducted with no external funding and is instead conducted with internal funding from the Department of Periodontology (Peking University School and Hospital of Stomatology).

\section{Authors' contributions}

$\mathrm{ZY}, \mathrm{JH}$, and $\mathrm{YZ}$ conceived the study design and participated in its coordination. ZY, JY and JH drafted the protocol. QL and HZ perform the interventions. YH, YY, LM, WH and CZ participated in the recruitment and allocation. The publication of the current study protocol was approved by all authors listed. All authors read and approved the final manuscript.

\section{Acknowledgments}

We would like to thank Xiang Yu (The Ohio State University, Columbus, OH, USA) for linguistic editing and proofreading.

\section{References}


1. Stuart HJ, Brian CO. Dental implant restoration: Principles and procedures. In: Stuart HJ, Brian CO, editors. Quintessence Publishing. The U.S.A.; 2011. p. 130-141.

2. Mohan N, Wolf J, Dym H. Maxillary sinus augmentation. Dent Clin N Am. 2015;59:375-388.

3. Lundgren L, Cricchio C, Hallman M, Jungner M, Rasmusson L, Sennerby L. Sinus floor elevation procedures to enable implant placement and integration: Techniques, biological aspects and clinical outcomes. Periodontology 2000. 2017;73:103-120.

4. Xingtian X, Zuolin W. Modern trend in lateral sinus floor augmentation. Journal of Oral and Maxillofacial Surgery. 2010;20:145-149.

5. Tan WC, Lang NP, Zwahlen M, Pjetursson BE. A systematic review of the success of sinus floor elevation and survival of implants inserted in combination with sinus floor elevation. Part Il: Transalveolar technique. J Clin Periodontol. 2008;35:241-254.

6. Falah M, Sohn DS, Srouji S. Graftless sinus augmentation with simultaneous dental implant placement: Clinical results and biological perspectives. Int J Oral Maxillofac Surg. 2016; 45:1147-53.

7. Felice P, Pistilli R, Piattelli M, et al. 1-stage versus 2-stage lateral sinus lift procedures: 1-year postloading results of a multicentre randomised controlled trial. Eur J Oral Implantol. 2014;7:65-75

8. Felice P, Pistilli R, Piattelli M, et al. 1-stage versus 2-stage lateral maxillary sinus lift procedures: 4month post-loading results of a multicenter randomised controlled trial. Eur $\mathrm{J}$ Oral Implantol. 2013;6:153-165

9. Khoury F, Keller P, Keeve P L. Stability of grafted implant placement sites after sinus floor elevation using a layering technique: 10 -Year clinical and radiographic results. Int J Oral Maxillofac Implants. 2017;32:1086-1096.

10. Dogan E, Dursun E, Tosun E, et al. Evaluation of hyaluronic matrix efficacy in sinus augmentation: $A$ randomized-controlled histomorphometric and micro-computed tomography analysis. Int J Oral Maxillofac Surg. 2017;46:931-937.

11. Beitlitum I, Habashi W, Tsesis I, et al. Extended maxillary sinus augmentation to the apical area of the neighboring teeth: Advantages and limitations. The International Journal of Periodontics \& Restorative Dentistry. 2018;38:451-456.

12. Tatum H Jr. Maxillary and sinus implant reconstructions. Dent Clin North Am. 1986;30:207-29

13. Kw F. VAS - Visuell analog skala. Tidsskr Nor Laegeforen. 2014;134:323.

14. Yalin Z, Wenjie H, Tao X, et al. Histomorphometric evaluation of ridge preservation after molar tooth extraction. Journal of Peking University (Health Sciences). 2017;49:169-175.

15. Pjetursson BE, Lang NP. Sinus floor elevation utilizing the transalveolar approach. Periodontology 2000. 2014;66:59-71.

16. Thoma DS, Zeltner M, Hüsler J, Hämmerle CH, Jung RE. EAO supplement working group 4 - EAO CC 2015: Short implants versus sinus lifting with longer implants to restore the posterior maxilla: A systematic review. Clin Oral Implants Res. 2015;26:s154-s169. 
17. Lee JE, Jin SH, Ko Y, et al. Evaluation of anatomical considerations in the posterior maxillae for sinus augmentation. World Journal of Clinical Cases. 2014;2:683-388.

18. Kao RT. Strategic extraction: A paradigm shift that is changing our profession. J Periodontol. 2008;79:971-7.

19. Lundgren D, Rylander H, Laurell L. To save or to extract, that is the question. Natural teeth or dental implants in periodontitissusceptible patients: Clinical decision-making and treatment strategies exemplified with patient case presentations. Periodontology 2000. 2008;47:27-50.

20. Morita K, Tsuka H, Kato K, et al. Factors related to masticatory performance in healthy elderly individuals. J Prosthodont Res. 2018;62:432-435.

21. Danesh-Sani SA, Loomer PM, Wallace SS. A comprehensive clinical review of maxillary sinus floor elevation: anatomy, techniques, biomaterials and complications. Br J Oral Maxillofac Surg. 2016;54:724-30.

22. Carrao V, Dematteis I. Maxillary sinus bone augmentation techniques. Oral Maxillofac Surg Clin North Am. 2015;27:245-53.

23. Stern A, Green J. Sinus lift procedures: An overview of current techniques. Dent Clin North Am. 2012;56:219-33.

24. Graetz C, Dorfer CE, Kahl M, Kocher T, et al. Retention of questionable and hopeless teeth in compliant patients treated for aggressive periodontitis. J Clin Periodontol. 2011;38:707-741.

25. Mcguire MK, Nunn ME. Prognosis versus actual outcome II: The effectiveness of clinical parameters in developing an accurate prognosis. J Periodontol. 1996;67:658-665.

\section{Figures}




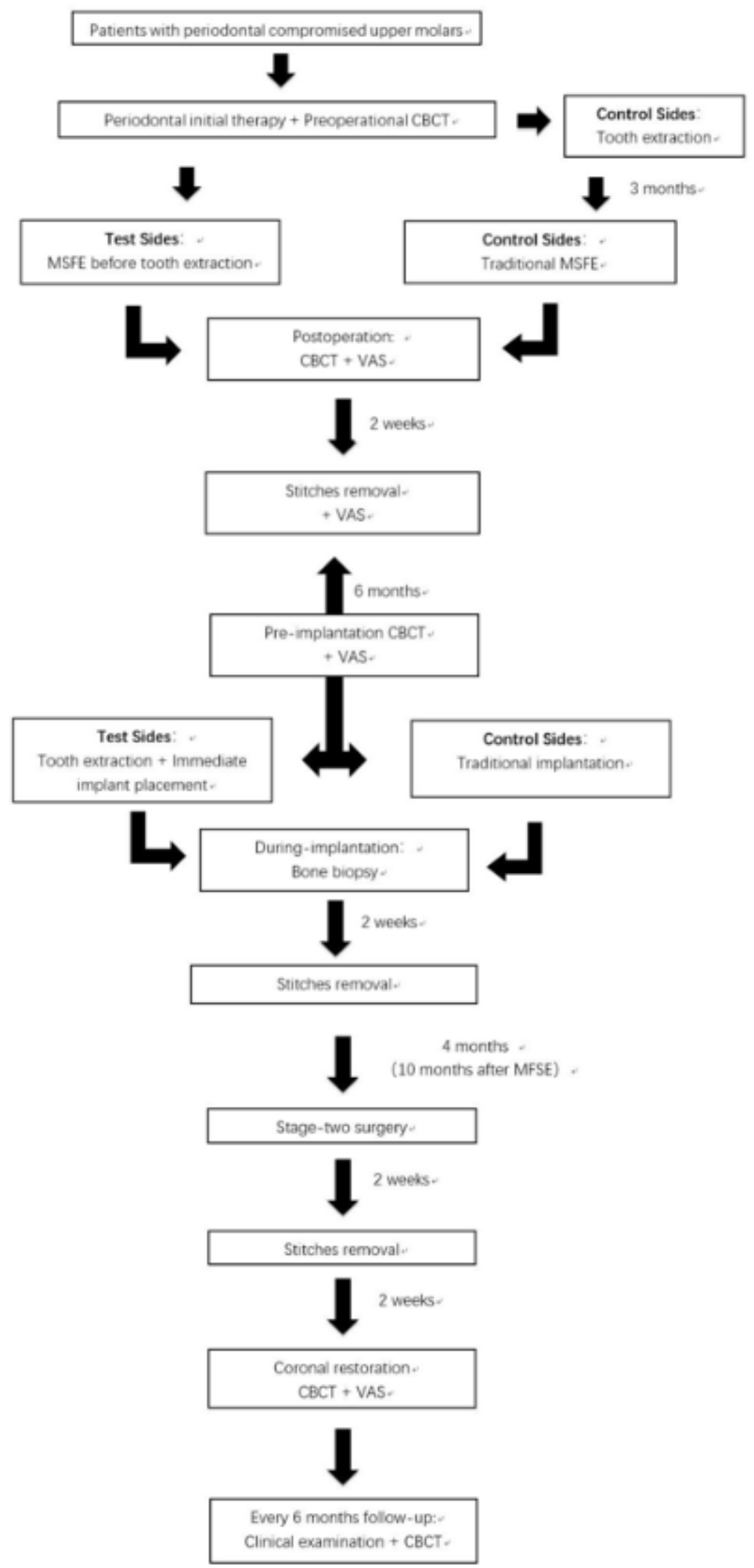

Figure 1

The flow of participants 


\begin{tabular}{|c|c|c|c|c|c|c|c|c|c|}
\hline \multirow[b]{3}{*}{ TIMEPOINT } & \multicolumn{9}{|c|}{ STUDY PERIOD } \\
\hline & \multirow{2}{*}{$\begin{array}{c}\text { Enrolment } \\
\text { Prior to } \\
\text { Allocation }\end{array}$} & \multirow{2}{*}{$\begin{array}{c}\text { Allocation } \\
\begin{array}{c}\text { Month } \\
0\end{array}\end{array}$} & \multicolumn{7}{|c|}{ Post-allocation } \\
\hline & & & $\begin{array}{c}\text { Month } \\
-3\end{array}$ & $\begin{array}{c}\text { Week } \\
2\end{array}$ & $\begin{array}{c}\text { Month } \\
6 \\
\end{array}$ & $\begin{array}{c}\text { Month } \\
10\end{array}$ & $\begin{array}{c}\text { Month } \\
12\end{array}$ & $\begin{array}{c}\text { Month } \\
18 \\
\end{array}$ & $\begin{array}{c}\text { Month } \\
24 \\
\end{array}$ \\
\hline \multicolumn{10}{|l|}{ ENROLMENT: } \\
\hline \multirow{2}{*}{$\begin{array}{l}\text { Eligibility screen } \\
\text { Informed consent }\end{array}$} & $\mathrm{x}$ & & & & & & & & \\
\hline & $\mathrm{X}$ & & & & & & & & \\
\hline Allocation & & $\mathrm{X}$ & & & & & & & \\
\hline \multicolumn{10}{|l|}{ INTERVENTIONS: } \\
\hline \multicolumn{10}{|l|}{$\begin{array}{r}\text { The traditional } \\
\text { MSFE }\end{array}$} \\
\hline $\begin{array}{r}\text { The modified } \\
\text { MSFE }\end{array}$ & & $\mathrm{X}$ & & $\leftarrow$ & & & & & \\
\hline \multicolumn{10}{|l|}{ ASSESSMENTS: } \\
\hline \multirow[t]{2}{*}{$\begin{array}{r}\text { Implant survival } \\
\text { rate }\end{array}$} & & & & & & $\mathrm{X}$ & $\mathrm{X}$ & $\mathrm{X}$ & $\mathrm{X}$ \\
\hline & & & & $\mathrm{X}$ & $\mathrm{X}$ & & $\mathrm{X}$ & & \\
\hline \multirow[t]{2}{*}{$\begin{array}{r}\text { Vertical bone } \\
\text { height }\end{array}$} & & $\mathrm{X}$ & & $\mathrm{X}$ & $\mathrm{X}$ & & $\mathrm{X}$ & $\mathrm{X}$ & $\mathrm{X}$ \\
\hline & & & & & $\mathrm{X}$ & & & & \\
\hline
\end{tabular}

Figure 2

SPIRIT figure

\section{Supplementary Files}

This is a list of supplementary files associated with this preprint. Click to download.

- AdditionalFile1SPIRIT2013Statement.doc

- AdditionalFile2ProtocolAmendmentsRecord.docx 